Preprint

UCRL-JC-137721

\title{
Molecular Dynamics Simulation of Cascade Damage in Gold
}

E. Alonso, M. J. Caturla, M. Tang, H. Huang, T. Diaz de la Rubia

This article was submitted to Materials Research Society Conference, Boston, MA., December 25,1996

\section{February 1, 2000}

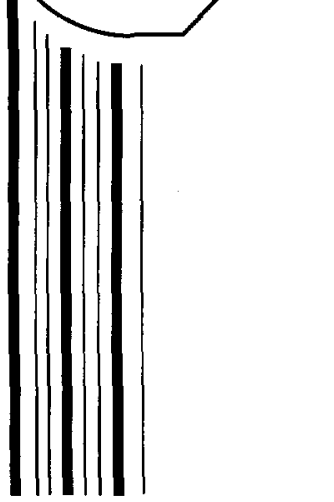




\section{DISCLAIMER}

This document was prepared as an account of work sponsored by an agency of the United States Government. Neither the United States Government nor the University of California nor any of their employees, makes any warranty, express or implied, or assumes any legal liability or responsibility for the accuracy, completeness, or usefulness of any information, apparatus, product, or process disclosed, or represents that its use would not infringe privately owned rights. Reference herein to any specific commercial product, process, or service by trade name, trademark, manufacturer, or otherwise, does not necessarily constitute or imply its endorsement, recommendation, or favoring by the United States Government or the University of California. The views and opinions of authors expressed herein do not necessarily state or reflect those of the United States Government or the University of California, and shall not be used for advertising or product endorsement purposes.

This is a preprint of a paper intended for publication in a journal or proceedings. Since changes may be made before publication, this preprint is made available with the understanding that it will not be cited or reproduced without the permission of the author.

This work was performed under the auspices of the United States Department of Energy by the University of California, Lawrence Livermore National Laboratory under contract No. W-7405-Eng-48.

This report has been reproduced directly from the best available copy.

Available electronically at http://www.doc.gov/bridge

Available for a processing fee to U.S. Department of Energy

And its contractors in paper from

U.S. Department of Energy

Office of Scientific and Technical Information

P.O. Box 62

Oak Ridge, TN 37831-0062

Telephone: (865) 576-8401

Facsimile: (865) 576-5728

E-mail: reports@adonis.osti.gov

Available for the sale to the public from

U.S. Department of Commerce

National Technical Information Service

5285 Port Royal Road

Springfield, VA 22161

Telephone: (800) 553-6847

Facsimile: (703) 605-6900

E-mail: orders@ntis.fedworld.gov

Online ordering: http://www.ntis.gov/ordering.htm

\section{OR}

Lawrence Livermore National Laboratory

Technical Information Department's Digital Library

http://www.llnl.gov/tid/Library.html 
for $45^{\circ}$ tilted incidence, both are he $\mathrm{Xe}^{+}$ions with $45^{\circ}$ tilted inci$\mathrm{ieV}$. Combining the doubling of that fig. 2 is valid for $5 \mathrm{keV}$ ions is with $1 \mathrm{ML}$ depths.

racancy islands with growing ion $\mathrm{Xe}^{+}$irradiation.

ıdiation can give detailed insight bardment. Within a model situaa.heters (ion fluence, target mass ıergy) could be attributed to indi1 surface diffusion. E. g., we can sertain temperature from the de-

One of the authors (K. P. R.) is

993).

!; Jülich, Germany, 1991; p. 15.

sr, New York, 1981; p. 179, 188,

(1991)

- Growth, Cambridge University

(1991).

:rosion depth for a fluence of ield) $\approx 1$ (cf. [9]), the amount of $1 \times 10^{16} \mathrm{Ar}^{+} \mathrm{cm}^{-2}$ fluence.

)4)

f Ions in Solids, Pergamon, New

\section{Molecular Dynamics Simulation of Cascade Damage in Gold}

E. Alonso, M.J. Caturla, M.Tang, H. Huang, and T. Diaz de la Rubia University of California, Lawrence Livermore National Laboratory, Livermore, CA 94551

Abstract

High-energy cascades have been simulated in gold using molecular dynamics with a modified embedded atom method potential. The results show that both vacancy and interstitial clusters form with high probability as a result of intracascade processes. The formation of clusters has been interpreted in terms of the high pressures generated in the core of the cascade during the early stages. We provide evidence that correlation between interstitial and vacancy clustering exists.

\section{Introduction}

The primary damage state in heavy ion, neutron or self- irradiated metals is a result of processes taking place within the lifetime of the displacement cascade. These cascades have been a subject of study for many years, and in particular, molecular dynamics (MD) simulations have been used extensively over the last ten years [1]. From the results of these MD studies, a relatively consistent picture of defect production has developed. Most significant, perhaps, is the fact that these simulations predict that even at low temperature both vacancies and interstitials can be produced either isolated or as defect clusters within the 1 to 100 ps of the cascade lifetime, i.e., without the assistance of significant thermal diffusion. The production of interstitials is due to long replacement collision sequences (RCSs) and other processes that result in clustering and dislocation loop formation directly in the periphery of the cascade. The vacancies appear in the core of the cascade region after resolidification of the melt and depending on the details of the cooling process and the properties of the irradiated material, can also be found in large clusters and Frank dislocation loops. Despite all these efforts however, no clear picture that relates the appearance of interstitial clusters with physical processes occurring within the cascade lifetime has emerged. Moreover, experimental validation of interstitial loop formation in cascades is hampered by the fact that TEM observations are limited by resolution considerations to clusters larger than some $10 \AA$ containing one to several hundred defects and use very thin foils from which small prismatic loops can easily escape. Shimomura et al [2] carried out liquid $\mathrm{He}$ irradiations of metals with $14 \mathrm{MeV}$ neutrons and in the thick sections of their samples observed loop growth during annealing below stage III after cold $(20 \mathrm{~K})$ transfer of the irradiated specimens to the TEM, indicating that interstitial-type dislocation loops were present in the sample after liquid He irradiation.

In this paper, we describe recent $\mathrm{MD}$ simulations of cascades in $\mathrm{Au}$ using an embedded atom method (EAM) potential for $\mathrm{Au}$ [3] that has been modified to account for short

$$
\text { MRS Symp. Pre v439, } 367 \text { (1997) }
$$


range interactions during the early stages of the cascade. The results are for 10 and $30 \mathrm{keV}$ cascades and generally show that a large fraction of the produced defects are in clusters. Only in one case, for a $10 \mathrm{keV}$ cascade, did we not find any clustering of the defects. In this case, the defect production efficiency was only about $6 \%$ of the NRT standard [4]. Moreover, in no case have we found cascades in which vacancy clustering occurs without interstitial clustering, or viceversa. We discuss the details of the cascade evolution and compare temperature, density and pressure profiles for a case where clusters were found to the case where no clustering took place. We show that the temperature profiles are similar for both cases, but that the hydrostatic and shear pressure profiles are substantially different.

\section{Method}

High-energy cascades in the bulk have been generated in gold using MD simulations with the MDCASK code. This code takes advantage of massively parallel computers, and the results were obtained on a 256 node Cray T3D. A fourth-order predictor-corrector algorithm was used to solve the equations of motion. Interatomic interactions were modeled using the EAM potential of Foiles et al [3]. The potential was modified by Ghaly et al. [5] to account for short range interaction. Periodic boundary conditions were indeed imposed and a damping function based on the Langevin equation was also included to prevent energy from reentering into the simulation box and to control the temperature. All calculations were performed at constant volume.

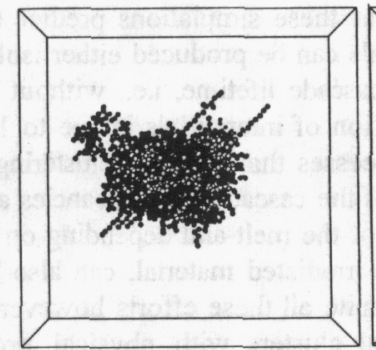

(a)

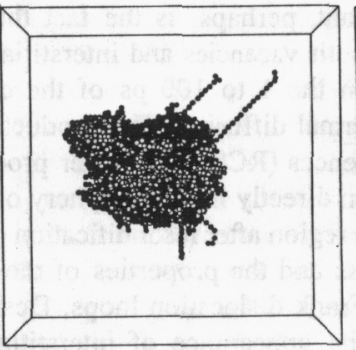

(b)

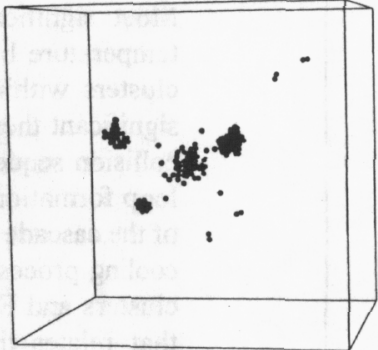

(c)

Figure 1. Three snapshots of the displaced atoms during a $10 \mathrm{keV}$ cascade in $\mathrm{Au}$. (a) 0.4 ps, (b) $2.0 \mathrm{ps}$, and (c) $27 \mathrm{ps}$. The interstitials (dark circles) are found mostly in clusters, as are the vacancies (light circles)

\section{Results anc}

Several sna final shape formation 0

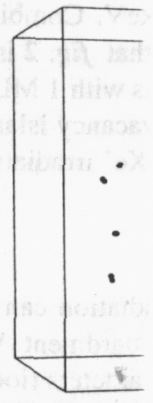

Figure 2 . from a 10 which no $\mathrm{c}$

Monte Car cluster forn the vacanci reach their :

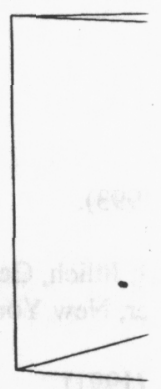

Figure 3. $\mathrm{P}$ a $30 \mathrm{keV} \mathrm{cc}$ of the vace are in clus 
he results are for 10 and $30 \mathrm{keV}$ roduced defects are in clusters. iny clustering of the defects. In it $6 \%$ of the NRT standard [4]. sancy clustering occurs without $s$ of the cascade evolution and case where clusters were found at the temperature profiles are 1 shear pressure profiles are

old using MD simulations with ely parallel computers, and the ourth-order predictor-corrector Interatomic interactions were he potential was modified by iodic boundary conditions were ,evin equation was also included and to control the temperature.

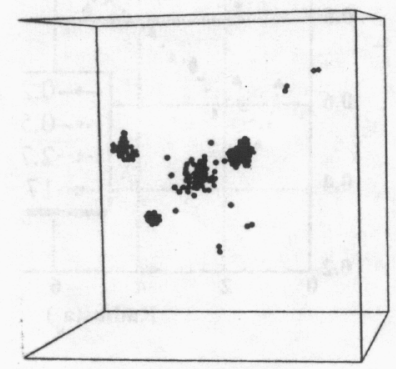

(c)

$10 \mathrm{keV}$ cascade in Au. (a) 0.4 s) are found mostly in clusters,

\section{Results and Discussion}

Several snapshots of a $10 \mathrm{keV}$ cascade at $50 \mathrm{~K}$ are displayed in Figure 1. Notice that the final shape of the cascade is already reached at $0.4 \mathrm{ps}$. At that point, the onset of the formation of the first cluster is clearly observed, as well as the beginning of the RCSs. The

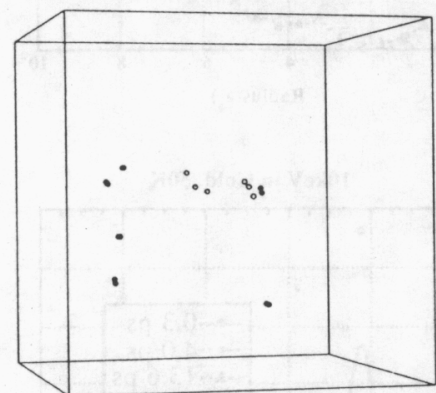

Figure 2. Defect configuration from a $10 \mathrm{keV}$ cascades in $\mathrm{Au}$ in which no clusters are formed maximum volume of the cascade is reached at about 2 ps. At 27 ps one can consider that the cascade has terminated, this value being the maximum time reached in our MD study. The final number of Frenkel pairs is 66 , i.e. $66 \%$ of the prediction of the NRT collisional model with a $40 \mathrm{eV}$ average threshold displacement energy. More importantly, 62 of them are in clusters. As is expected in metals, the vacancies are distributed in the central core of the cascade while the interstitials are located in the outer part. Most of the vacancies (55) form one big cluster. We also observe a trend in this cluster to evolve to a dislocation loop. This last issue can be checked by extending the simulation time by MD, which would be a tedious task, or coupling MD with force-bias Monte Carlo. The sizes of the interstitial clusters are variable $(34,20,8)$. The smallest cluster forms a prismatic dislocation loop and the larger ones exhibit the same tendency as the vacancies. Then, the same procedure could be applied to make sure whether they reach their minimum energy configuration as a loop or not.

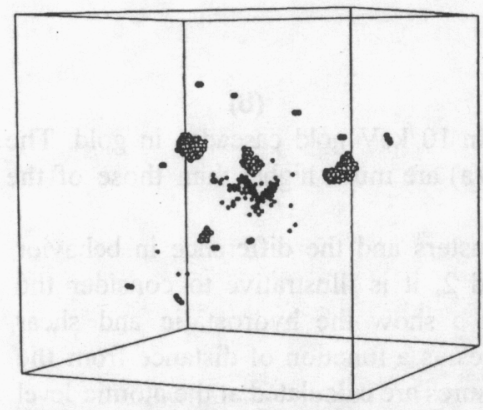

Figure 3. Primary damage state from a $30 \mathrm{keV}$ cascade in Au. The majority of the vacancies and the interstitials are in clusters, as discussed in the text.
Figure 2 shows the final defect configuration generated by another $10 \mathrm{keV}$ cascade at $300 \mathrm{~K}$. In this case, no clusters are observed and the defect production efficiency is only $6 \%$ of the NRT prediction. All the interstitials are ejected by RCSs. In figure 3 , we show the primary damage state at 60 ps resulting from a $30 \mathrm{keV}$ cascade at $300 \mathrm{~K}$. Here, the defect production efficiency is $30 \%$ of NRT (112 Frenkel pairs) and about $70 \%$ of the defects are in clusters. About 70 vacancies are clustered in two Frank loops. Only 9 interstitials remain as single type and only 2 as diinterstitials. The rest are present in four clusters of sizes $36,33,25$ and 7 . 

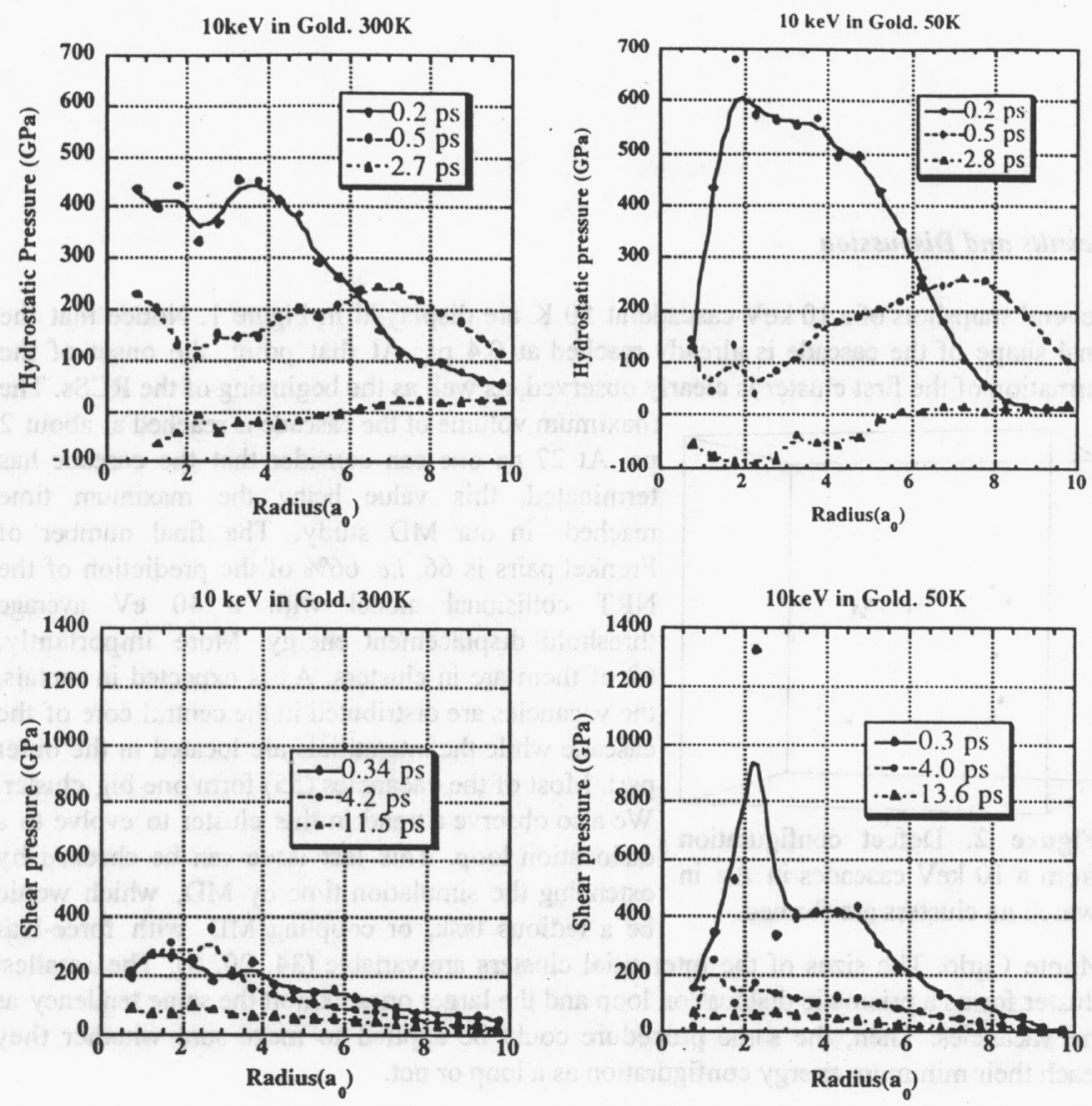

(a)

(b)

Figure 4. Hydrostatic and shear pressure profiles in $10 \mathrm{keV}$ gold cascades in gold. The peak values for the cascade with cluster formation (a) are much higher than those of the cascade without clusters (b).

In order to understand the production of defect clusters and the difference in behavior between the two $10 \mathrm{keV}$ cascades of figures 1 and 2, it is illustrative to consider the details of the cascade evolution. Figures $4 \mathrm{a}$ and $\mathrm{b}$ show the hydrostatic and shear pressure profiles calculated for both $10 \mathrm{keV}$ cascades as a function of distance from the centroid of energy distribution of each cascade. Pressures are calculated at the atomic level according to the formulation of Vitek et al [6]. At short times (around $0.2 \mathrm{ps),} \mathrm{when} \mathrm{the}$ cluster formation is about to take place, the pressure generated in the first cascade is much higher than in the second one. The extremely large values of the pressures are due to the fact that the calculations are carried out by considering the volume derivatives of the energy of each atom. Thus, th times, the region is not yet the and are in close proximity to distribution of collision event establishment of extremely hig collisions are responsible for se clustering. Note also that as sh the same time as the RCSs. ( cascade region there is a drasti Figures $5 \mathrm{a}$ and $\mathrm{b}$ where radial $\mathrm{d}$ cascade that results in defect cl than for the cascade that does 1 significant difference in the tem any time.

\section{Conclusions}

We performed MD simulatio phenomena. Our results indica short simulation times $(0.2 \mathrm{~F}$ correlation between interstitia

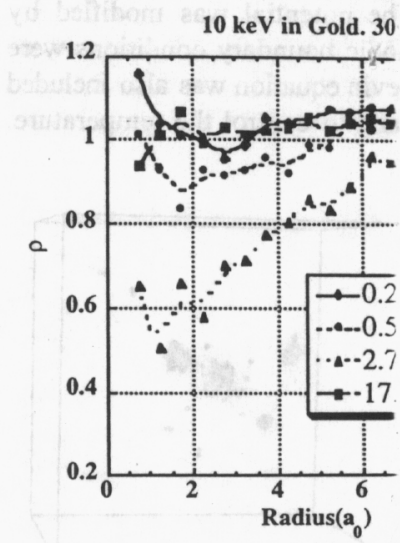

(a)

Figure 5. Radial density profile: is reduced by a factor two in much higher in the cascade with small times. 


\section{$10 \mathrm{keV}$ in Gold. $50 \mathrm{~K}$}

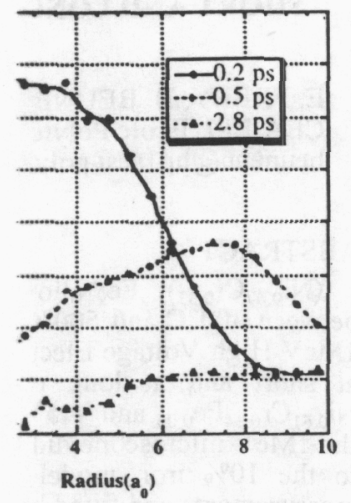

$10 \mathrm{keV}$ in Gold. 50K

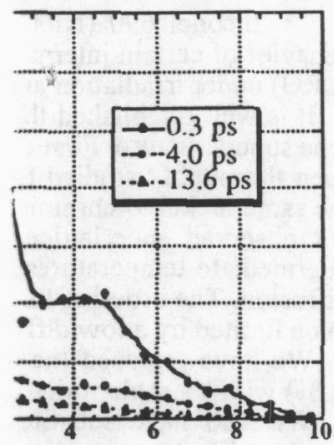

Radius(a)

(b)

gold cascades in gold. The th higher than those of the

the difference in behavior llustrative to consider the he hydrostatic and shear ction of distance from the Iculated at the atomic level (around $0.2 \mathrm{ps}$ ), when the in the first cascade is much e pressures are due to the volume derivatives of the energy of each atom. Thus, the results are skewed by the fact that during these early times, the region is not yet thermalized and many atoms have very high kinetic energies and are in close proximity to other atoms. Nevertheless, the results show that the distribution of collision events during the very early stages of the cascade and the establishment of extremely high pressure gradients as a result of localized high energy collisions are responsible for setting up the conditions for both vacancy and interstitial clustering. Note also that as shown in Fig. 1 the interstitial clusters are ejected at about the same time as the RCSs. Concomitant with the high pressures established in the cascade region there is a drastic reduction in the atomic density. This can be seen in Figures $5 \mathrm{a}$ and $\mathrm{b}$ where radial density profiles at various times are shown. Again, for the cascade that results in defect clustering, the density reduction at early times is much larger than for the cascade that does not produce clusters. It is also interesting to note that no significant difference in the temperature profiles for these two cascades were observed at any time.

\section{Conclusions}

We performed MD simulations in the bulk and we analyzed interstitial clustering phenomena. Our results indicate that the tremendous increase in pressure occurring at short simulation times $(0.2 \mathrm{ps})$ is responsible for the cluster formation. A strong correlation between interstitial and vacancy clustering can be extracted from our

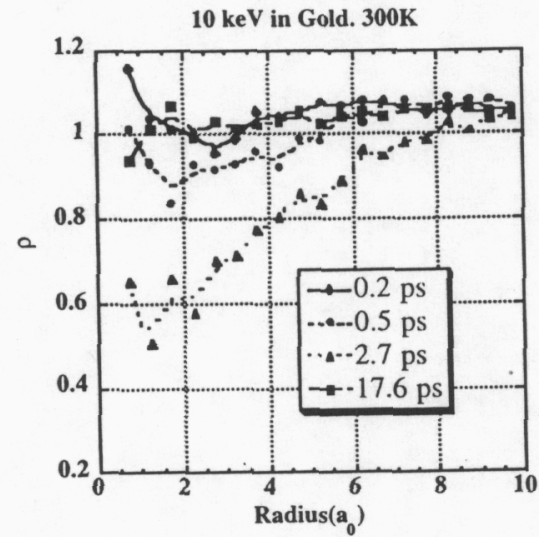

(a)

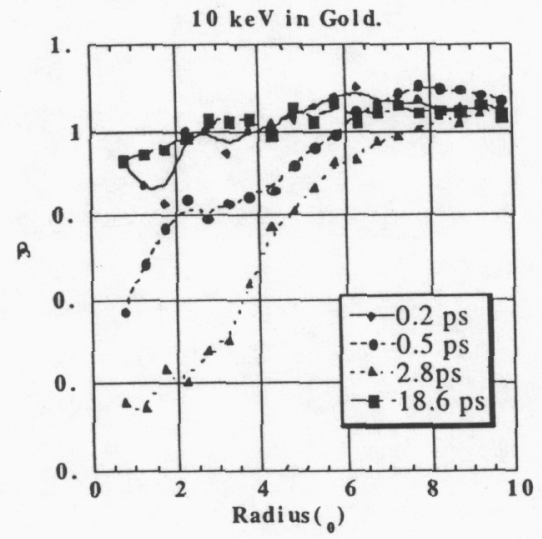

(b)
Figure 5. Radial density profiles of $10 \mathrm{keV}$ gold cascades in gold. After $2.7 \mathrm{ps,} \mathrm{the} \mathrm{density}$ is reduced by a factor two in the cascade without clusters (a). The volume reduction is much higher in the cascade with cluster formation (b) due to the more elevated pressures at small times. 
simulations as well as a higher efficiency for cascades where clusters are present. Our results allowed us to extract valuable information on diffusivities to be used in future kinetic-Monte Carlo studies. Ongoing calculations will extend the time scale achieved through MD by using force bias Monte Carlo.

\section{Acknowledgements}

This work was performed under the auspices of the US Department of Energy by Lawrence Livermore National Laboratory under contract No. W-7405-Eng-48.

\section{References}

1 T. Diaz de la Rubia, Ann. Rev. Mater. Sci. 26, 613 (1996)

2. Y. Shimomura, H. Fukushima, and M.W. Guinan, J. Nucl. Mater. 174, 210 (1990)

3 S.M. Foiles, M.I Baskes and M.S. Daw, Phys. Rev. B 33 (1986) 7983-7991

4 M.J. Norgett, M.T. Robinson and I.M. Torrens, Nuc. Eng. and Design 33 (1975) 50-54

5. M. Ghaly, Private Communication

6 V. Vitek and T. Egami, Phys. Stat. Sol. B 144 (1987) 145-156 